\title{
Checklist of the families Scathophagidae, Fanniidae and Muscidae of Finland (Insecta, Diptera)
}

\author{
Jere Kahanpää', Antti Haarto² \\ I Finnish Museum of Natural History, Zoology Unit, P.O. Box 17, FI-00014 University of Helsinki, Finland \\ 2 Zoological Museum, Section of Biodiversity and Environmental Science, Department of Biology, University \\ of Turku, FI-20014 Turku, Finland
}

Corresponding author:Jere Kahanpää (jere.kahanpaa@helsinki.fi)

Academic editor: J. Salmela | Received 5 February 2014 | Accepted 19 May 2014 | Published 19 September 2014

http://zoobank.org/776A4380-DB69-43C6-96AA-B6E336E68E1B

Citation: Kahanpää J, Haarto A (2014) Checklist of the families Scathophagidae, Fanniidae and Muscidae of Finland (Insecta, Diptera). In: Kahanpää J, Salmela J (Eds) Checklist of the Diptera of Finland. ZooKeys 441: 347-367. doi: $10.3897 /$ zookeys.441.7142

\begin{abstract}
A revised checklist of the Scathophagidae, Fanniidae and Muscidae recorded from Finland is presented. Phaonia amicula Villeneuve, 1922 is noted from Finland for the first time.
\end{abstract}

\section{Keywords}

Species list, Finland, Diptera, biodiversity, faunistics

\section{Introduction}

Four families make up the traditional superfamily Muscoidea: Scathophagidae, Anthomyiidae, Fanniidae and Muscidae. The monophyly of the superfamily has been strongly questioned (Nirmala et al. 2001, Kutty et al. 2010) on the basis of DNA sequence analyses.

Three of the four families of Muscoidea are treated in this paper, the fourth (Anthomyiidae) is covered in a separate paper in this issue of ZooKeys. All three are important components of the Finnish fly fauna as can be seen from the large number of species involved and the fact that the species recorded from the country represent up to one fifth of the world fauna in the case of Scathophagidae. 
Table I. Number of species by family.

\begin{tabular}{c|c|c|c|c}
\hline \multirow{2}{*}{ Family } & \multicolumn{3}{|c|}{ Number of species in } & \multirow{2}{*}{ Level of knowledge } \\
\cline { 2 - 5 } & World (Pape et al. 2011) & Europe & Finland & good \\
\hline Scathophagidae & 419 & 158 & $85-86$ & average \\
\hline Fanniidae & 359 & 83 & 61 & average \\
\hline Muscidae & 5218 & 572 & $307-309$ & \\
\hline
\end{tabular}

Unfortunately the muscoid flies have been poorly collected and studied in Finland in comparison with many other Diptera families. Lauri Tiensuu concentrated on these families during the 1930s $(1935,1938,1939)$ and Walter Hackman (1956) reviewed the Finnish Scathophagidae. During the last ten years, both the fanniids (Kahanpää and Haarto 2013) and the muscids (Kahanpää 2013) have attracted some attention in the country. The number of species known from the country has increased markedly since the year 2000 .

The Finnish muscoid species were last listed by Hackman (1980).

\section{Checklist}

suborder Brachycera Macquart, 1834

clade Eremoneura Lameere, 1906

clade Cyclorrhapha Brauer, 1863

infraorder Schizophora Becher, 1882

clade Muscaria Enderlein, 1936

parvorder Calyptratae Robineau-Desvoidy, 1830

superfamily Muscoidea Latreille, 1802

SCATHOPHAGIDAE Robineau-Desvoidy, 1830

DELININAE Séguy, 1952

DELINA Robineau-Desvoidy, 1830

Delina nigrita (Fallén, 1819)

LEPTOPA Zetterstedt, 1838

Leptopa filiformis Zetterstedt, 1838

MICROPSELAPHA Becker, 1894

Micropselapha filiformis (Zetterstedt, 1846)

PARALLELOMMA Strobl, 1894

= Chylizosoma Hendel, 1924

Parallelomma medium Becker, 1894

? Parallelomma paridis Hering, 1923 (see Notes)

Parallelomma sellatum (Hackman, 1956)

Parallelomma vittatum (Meigen, 1826)

PHROSIA Robineau-Desvoidy, 1830 
Phrosia albilabris (Fabricius, 1805)

SCATHOPHAGINAE Robineau-Desvoidy, 1830

ACANTHOCNEMA Becker, 1894

sg. Clinoceroides Hendel, 1917

Acanthocnema glaucescens (Loew, 1864)

= nigripes Ringdahl, 1936

ACEROCNEMA Becker, 1894

Acerocnema macrocera (Meigen, 1826)

= tiefi Becker, 1894

= pokornyi Becker, 1894

ALLOMYELLA Malloch, 1923

Allomyella albipennis (Zetterstedt, 1838)

Allomyella frigida (Holmgren, 1883)

= portenkoi (Stackelberg, 1952) misid.

BOSTRICHOPYGA Becker, 1894

Bostrichopyga crassipes (Zetterstedt, 1838)

CHAETOSA Coquillett, 1898

Chaetosa punctipes (Meigen, 1826)

CLEIGASTRA Macquart, 1835

= Cnemopogon Rondani, 1856

Cleigastra apicalis (Meigen, 1826)

CONISTERNUM Strobl, 1894

= Coniosternum Becker, 1894

Conisternum lapponicum (Ringdahl, 1920)

Conisternum obscurum (Fallén, 1819)

Conisternum tinctinerve (Becker, 1894)

CORDILURA Fallén, 1810

= Cordylura Meigen, 1826 emend.

sg. Cordilura Fallén, 1810

Cordilura aberrans Becker, 1894

Cordilura aemula Collin, 1958

Cordilura atrata Zetterstedt, 1846

Cordilura ciliata Meigen, 1826

Cordilura picipes Meigen, 1826

Cordilura picticornis Loew, 1864

Cordilura proboscidea Zetterstedt, 1838

Cordilura pubera (Linnaeus, 1758)

Cordilura pudica Meigen, 1826

Cordilura rufimana Meigen, 1826

Cordilura socialis (Becker, 1894)

= freyi Hackman, 1956

sg. Cordilurina James, 1955

= Parallelomma Becker, 1894 preocc. 
Cordilura albipes Fallén, 1819

Cordilura fuscipes Zetterstedt, 1838

sg. Scoliaphleps Becker, 1894

Cordilura ustulata Zetterstedt, 1838

= melanacra Loew, 1873

COSMETOPUS Becker, 1894

Cosmetopus dentimana (Zetterstedt, 1838)

= fulvipes (Zetterstedt, 1838)

Cosmetopus longa (Walker, 1849)

= bergrothi Becker, 1900 in part

= fulvipes auct. nec (Zetterstedt, 1838)

Cosmetopus ringdahli Andersson, 1974

= bergrothi Becker, 1900 in part

ERNONEURA Becker, 1894

Ernoneura argus (Zetterstedt, 1838)

GONARCTICUS Becker, 1894

Gonarcticus abdominalis (Zetterstedt, 1846)

GONATHERUS Rondani, 1856

Gonatherus planiceps (Fallén, 1826)

GIMNOMERA Rondani, 1866

= Cochliarium Becker, 1894

Gimnomera albipila (Zetterstedt, 1846)

Gimnomera cuneiventris (Zetterstedt, 1846)

Gimnomera dorsata (Zetterstedt, 1838)

Gimnomera hirta Hendel, 1930

Gimnomera tarsea (Fallén, 1819)

HEXAMITOCERA Becker, 1894

Hexamitocera loxoceratum (Fallén, 1826)

HYDROMYZA Fallén, 1823

Hydromyza livens (Fabricius, 1794)

MEGAPHTHALMA Becker, 1894

Megaphthalma pallida (Fallén, 1819)

MEGAPHTHALMOIDES Ringdahl, 1936

Megaphthalmoides unilineatus (Zetterstedt, 1838)

MICROPROSOPA Strobl, 1894

Microprosopa haemorrhoidalis (Meigen, 1826)

Microprosopa lacteipennis Ringdahl, 1920

Microprosopa lineata (Zetterstedt, 1838)

Microprosopa pallidicauda (Zetterstedt, 1838)

NANNA Strobl, 1894

= Amaurosoma Becker, 1894

Nanna armillata (Zetterstedt, 1846)

Nanna articulata (Becker, 1894) 
Nanna bispinosa (Malloch, 1920)

Nanna brevifrons (Zetterstedt, 1838)

Nanna fasciata (Meigen, 1826)

Nanna flavipes (Fallén, 1819)

$=$ minuta $($ Becker, 1894)

$?=$ multisetosa (Hackman, 1956)

Nanna inermis (Becker, 1894)

Nanna leucostoma (Zetterstedt, 1846)

Nanna tibiella (Zetterstedt, 1838)

$=$ nigripes $($ Zetterstedt, 1846)

NORELLIA Robineau-Desvoidy, 1830

Norellia tipularia (Fabricius, 1794) (see Notes)

NORELLISOMA Hendel, 1910

Norellisoma lituratum (Wiedemann, 1826)

Norellisoma spinimanum (Fallén, 1819)

OKENIELLA Hendel, 1907

Okeniella caudata (Zetterstedt, 1838)

Okeniella dasyprocta (Loew, 1864)

ORTHACHETA Becker, 1894

Orthacheta pilosa (Zetterstedt, 1838)

PLEUROCHAETELLA Vockeroth, 1965

Pleurochaetella simplicipes (Becker, 1900)

POGONOTA Zetterstedt, 1860

sg. Pogonota Zetterstedt, 1860

Pogonota barbata (Zetterstedt, 1838)

sg. Lasioscelus Becker, 1894

Pogonota immunda (Zetterstedt, 1838)

= clavatus (Zetterstedt, 1846)

Pogonota sahlbergi Becker, 1900

SCATHOPHAGAMeigen, 1803

= Scatophaga Frabricius, 1805 emend.

= Scopeuma Meigen, 1800 suppr.

= Scatomyza Fallén, 1810

Scathophaga apicalis Curtis in Ross, 1835

Scathophaga furcata (Say, 1823)

Scathophaga incola Becker, 1900

Scathophaga inquinata (Meigen, 1826)

= analis Meigen, 1826

Scathophaga litorea (Fallén, 1819)

Scathophaga lutaria (Fabricius, 1794)

Scathophaga obscurinervis Becker, 1900

Scathophaga pictipennis Oldenberg, 1923

Scathophaga scybalaria (Linnaeus, 1758) 
Scathophaga stercoraria (Linnaeus, 1758)

Scathophaga suilla (Fabricius, 1794)

SPAZIPHORA Rondani, 1856

Spaziphora hydromyzina (Fallén, 1819)

= fascipes (Becker, 1894)

STAEGERIA Rondani, 1856

Staegeria kunzei (Zetterstedt, 1821)

TRICHOPALPUS Rondani, 1856

Trichopalpus fraternus (Meigen, 1826)

Trichopalpus nigribasis Curran, 1927

= pilirostris (Ringdahl, 1936)

Trichopalpus obscurellus (Zetterstedt, 1846)

= subarcticus (Ringdahl, 1936)

\section{FANNIIDAE Schnabl \& Dziezicki, 1911}

FANNIA Robineau-Desvoidy, 1830

= Homalomyia Bouché, 1834

= Coelomyia Haliday, 1840

Fannia aethiops Malloch, 1913

Fannia alpina Pont, 1970

Fannia armata (Meigen, 1826)

Fannia atra (Stein, 1895)

Fannia canicularis (Linnaeus, 1761)

Fannia carbonaria (Meigen, 1826)

Fannia carbonella (Stein, 1895)

Fannia coracina (Loew, 1873)

Fannia corvina (Verrall, 1892)

= halterata Ringdahl, 1918

Fannia cothurnata (Loew, 1873)

Fannia difficilis (Stein, 1895)

Fannia fuscitibia Stein, 1920

Fannia fuscula (Fallén, 1825)

Fannia genualis (Stein, 1895)

Fannia gotlandica Ringdahl, 1926

Fannia hirticeps (Stein, 1892)

Fannia hirundinis Ringdahl, 1948

Fannia immutica Collin, 1939

Fannia incisurata (Zetterstedt, 1838)

Fannia latifrontalis Hennig, 1955

Fannia latipalpis (Stein, 1892)

Fannia lepida (Wiedemann, 1817)

= mutica (Zetterstedt, 1845)

Fannia leucosticta (Meigen, 1838) 
Fannia limbata (Tiensuu, 1938)

= berolinensis Hennig, 1955

Fannia lucidula (Zetterstedt, 1860)

= glaucescens auct. nec (Zetterstedt, 1845)

Fannia lugubrina (Zetterstedt, 1838)

Fannia lustrator (Harris, 1780)

= hamata (Macquart, 1835)

Fannia manicata (Meigen, 1826)

Fannia melania (Dufour, 1839)

$=$ ciliata $($ Stein, 1895)

Fannia metallipennis (Zetterstedt, 1838)

= kowarzi (Verrall, 1892)

Fannia minutipalpis (Stein, 1895)

Fannia mollissima (Haliday, 1840)

= spathulata (Zetterstedt, 1845)

Fannia monilis (Haliday, 1838)

Fannia pallitibia (Rondani, 1866)

Fannia parva (Stein, 1895)

Fannia pauli Pont, 1997

$=$ nitida $($ Stein, 1895) preocc.

Fannia polychaeta (Stein, 1895)

Fannia postica (Stein, 1895)

Fannia posticata (Meigen, 1826)

$=$ pretiosa $($ Schiner, 1862)

Fannia rabdionata Karl, 1940

Fannia ringdablana Collin, 1939

= umbrosa auct. nec (Stein, 1895)

Fannia rondanii (Strobl, 1893)

= carbonaria (Rondani, 1871) preocc.

$=$ aerea misid.

Fannia scalaris (Fabricius, 1794)

= subscalaris Zimin, 1946

Fannia serena (Fallén, 1825)

Fannia similis (Stein, 1895)

Fannia slovaca Gregor \& Rozkošný, 2005

Fannia sociella (Zetterstedt, 1845)

Fannia spathiophora Malloch 1918

= nodulosa Ringdahl, 1926

Fannia speciosa (Villeneuve, 1898)

Fannia stigi Rognes, 1982

Fannia subatripes d'Assis-Fonseca, 1967

Fannia subpellucens (Zetterstedt, 1845)

Fannia subpubescens Collin, 1958 
Fannia tuberculata (Zetterstedt, 1849)

Fannia umbratica Collin, 1939

Fannia umbrosa (Stein, 1895)

= subumbros $a$ Ringdahl, 1934

Fannia verrallii (Stein, 1895)

Fannia vesparia (Meade, 1891)

Fannia vespertilionis Ringdahl, 1934

PIEZURA Rondani, 1844

= Platycoenosia Strobl, 1894

Piezura graminicola (Zetterstedt, 1846)

= boletorum (Rondani, 1866)

= mikii (Strobl, 1894)

Piezura pardalina (Rondani, 1866)

= graminicola auct. nec (Zetterstedt, 1846)

\section{MUSCIDAE Latreille, 1802}

ACHANTHIPTERINAE Hennig, 1962

ACHANTHIPTERA Rondani, 1856

Achanthiptera rohrelliformis (Robineau-Desvoidy, 1830)

= inanis (Fallén, 1825) preocc.

COENOSIINAE Verrall, 1888

tribe Coenosiini Verrall, 1888

COENOSIA Meigen, 1826

= Caricea Robineau-Desvoidy, 1830

= Oplogaster Rondani, 1856

= Dexiopsis Pokorny, 1893

Coenosia acuminata Strobl, 1898

= annulipes Ringdahl, 1932

Coenosia agromyzina (Fallén, 1825)

Coenosia ambulans Meigen, 1826

Coenosia bilineella (Zetterstedt, 1838)

Coenosia campestris (Meigen, 1830)

= sexnotata auct. nec Meigen, 1826

? Coenosia comita (Huckett, 1936) (see Notes)

= ovulifera Tiensuu, 1938

Coenosia dealbata (Zetterstedt, 1838)

= fulvicornis (Zetterstedt, 1845)

Coenosia flavimana (Zetterstedt, 1845)

= albatella (Zetterstedt, 1849)

Coenosia humilis Meigen, 1826

Coenosia intermedia (Fallén, 1825)

Coenosia lacteipennis (Zetterstedt, 1845)

Coenosia lineatipes (Zetterstedt, 1845) 
= albicornis misid.

Coenosia means Meigen, 1826

Coenosia mollicula (Fallén, 1825)

Coenosia octopunctata (Zetterstedt, 1838)

Coenosia paludis Tiensuu, 1939

Coenosia pedella (Fallén, 1825)

= decipiens Meigen, 1826

Coenosia perpusilla Meigen, 1826

Coenosia pudorosa Collin, 1953

Coenosia pulicaria (Zetterstedt, 1845)

Coenosia pumila (Fallén, 1825)

Coenosia pygmaea (Zetterstedt, 1845)

Coenosia ruficornis Macquart, 1835

= litoralis (Zetterstedt, 1846)

Coenosia rufipalpis Meigen, 1826

= flavicauda Ringdahl, 1937

Coenosia sallae Tiensuu, 1938

Coenosia testacea (Robineau-Desvoidy, 1830)

= tricolor (Zetterstedt, 1845)

= alleni $\mathrm{d}$ 'Assis-Fonseca, 1966

Coenosia tigrina (Fabricius, 1775)

Coenosia trilineella (Zetterstedt, 1838)

$=$ trilineata emend.

Coenosia verralli Collin, 1953

= steini Verrall, 1912 preocc.

LIMNOSPILA Schnabl, 1902

Limnospila albifrons (Zetterstedt, 1849)

LISPOCEPHALA Pokorny, 1893

Lispocephala alma (Meigen, 1826)

Lispocephala erythrocera (Robineau-Desvoidy, 1830)

Lispocephala falculata Collin, 1963

Lispocephala fuscitibia Ringdahl, 1944

Lispocephala pallipalpis (Zetterstedt, 1845)

Lispocephala spuria (Zetterstedt, 1838)

= vitripennis Ringdahl, 1951

Lispocephala verna (Fabricius, 1794)

MACRORCHIS Rondani, 1877

Macrorchis meditata (Fallén, 1825)

PSEUDOCOENOSIA Stein, 1916

Pseudocoenosia abnormis Stein, 1916

Pseudocoenosia solitaria (Zetterstedt, 1838)

= longicauda (Zetterstedt, 1860)

SCHOENOMYZA Haliday, 1833 
Schoenomyza litorella (Fallén, 1823)

tribe Limnophoriini Villeneuve, 1902

LIMNOPHORA Robineau-Desvoidy, 1830

Limnophora nigripes (Robineau-Desvoidy, 1830)

Limnophora pandellei Séguy 1923 (see Notes)

Limnophora riparia (Fallén, 1824)

Limnophora rotundata Collin, 1930

Limnophora tigrina (Am Stein, 1860)

$=$ notata (Fallén, 1823) preocc.

Limnophora triangula (Fallén, 1825)

Limnophora uniseta Stein, 1916

LISPE Latreille, 1796

Lispe consanguinea Loew, 1858

Lispe hydromyzina Fallén, 1825

Lispe litorea Fallén, 1825

Lispe melaleuca Loew, 1847

Lispe pygmaea Fallén, 1825

Lispe tentaculata (De Geer, 1776)

Lispe uliginosa Fallén, 1825

SPILOGONA Schnabl, 1911

Spilogona aerea (Fallén, 1825)

Spilogona albisquama (Ringdahl, 1932)

Spilogona alpica (Zetterstedt, 1845)

Spilogona arenosa (Ringdahl, 1918)

Spilogona atrisquamula Hennig, 1959

Spilogona baltica (Ringdahl, 1918)

Spilogona brunneifrons Ringdahl, 1931

Spilogona brunneisquama (Zetterstedt, 1845)

Spilogona carbonella (Zetterstedt, 1845)

Spilogona contractifrons (Zetterstedt, 1838)

Spilogona denigrata (Meigen, 1826)

Spilogona depressiuscula (Zetterstedt, 1838)

Spilogona depressula (Zetterstedt, 1845)

Spilogona dispar (Fallén, 1823)

= funeralis Rondani, 1866

Spilogona falleni Pont, 1984

= litorea auct. nec (Fallén, 1823)

Spilogona krogerusi (Ringdahl, 1941)

$=$ micans misid.

Spilogona leucogaster (Zetterstedt, 1838)

Spilogona malaisei (Ringdahl, 1920)

Spilogona marginifera Hennig, 1959

= marginalis (Fallén, 1824) preocc. 
Spilogona meadei (Schnabl, 1915)

Spilogona megastoma (Boheman, 1866)

Spilogona nigriventris (Zetterstedt, 1845)

Spilogona nitidicauda (Schnabl, 1911)

Spilogona novemmaculata (Zetterstedt, 1860)

Spilogona opaca (Schnabl, 1915)

Spilogona pacifica (Meigen, 1826)

= vana (Zetterstedt, 1845)

Spilogona palmeni (Ringdahl, 1935)

Spilogona pseudodispar (Frey, 1915)

= spinitibia $($ Ringdahl, 1918)

Spilogona pusilla (Huckett, 1932)

Spilogona quinquelineata (Zetterstedt, 1838)

Spilogona semiglobosa (Ringdahl, 1916)

Spilogona sororcula (Zetterstedt, 1845)

$=$ zetterstedti $($ Ringdahl, 1918) preocc.

Spilogona spectabilis (Tiensuu, 1938)

Spilogona surda (Zetterstedt, 1845)

Spilogona tenuis Hennig, 1959

Spilogona tornensis (Ringdahl, 1926)

Spilogona triangulifera (Zetterstedt, 1838)

Spilogona trianguligera (Zetterstedt, 1838)

= insularis (Collin, 1921)

Spilogona trigonata (Zetterstedt, 1838)

Spilogona tundrae (Schnabl, 1915)

= macropyga $($ Frey, 1915)

Spilogona tundrica (Schnabl \& Dziedzicki, 1911)

Spilogona varsaviensis (Schnabl \& Dziedzicki, 1911)

$=$ glauca $($ Stein, 1916)

Spilogona veterrima (Zetterstedt, 1845)

VILLENEUVIA Schnabl \& Dziedzicki, 1911

Villeneuvia aestuum (Villeneuve, 1902)

AZELIINAE Robineau-Desvoidy, 1830

tribe Azeliini Robineau-Desvoidy, 1830

AZELIA Robineau-Desvoidy, 1830

Azelia aterrima (Meigen, 1826)

Azelia cilipes (Haliday, 1838)

Azelia gibbera (Meigen, 1826)

Azelia monodactyla Loew, 1874

Azelia nebulosa Robineau-Desvoidy, 1830

= macquarti (Staeger in Schiødte, 1843)

Azelia trigonica Hennig, 1956

= nuda Hennig, 1956 
Azelia triquetra (Wiedemann, 1817)

Azelia zetterstedtii Rondani, 1866

DRYMEIA Meigen, 1826

= Pogonomyia Rondani, 1871

= Trichopticoides Ringdahl, 1931

Drymeia hamata (Fallén, 1823)

Drymeia tetra (Meigen, 1826)

Drymeia vicana (Harris, 1780)

$=\operatorname{decolor}($ Fallén, 1824)

HYDROTAEA Robineau-Desvoidy, 1830

= Ophyra Robineau-Desvoidy, 1830

= Lasiops Meigen, 1838

Hydrotaea aenescens (Wiedemann, 1830)

Hydrotaea albipuncta (Zetterstedt, 1845)

Hydrotaea anxia (Zetterstedt, 1838)

= bispinosa (Zetterstedt, 1845)

Hydrotaea armipes (Fallén, 1825)

= occulta (Meigen, 1826)

Hydrotaea basdeni Collin, 1939

Hydrotaea borussica Stein, 1899

Hydrotaea cyrtoneurina (Zetterstedt, 1845)

Hydrotaea dentipes (Fabricius, 1805)

Hydrotaea diabolus (Harrris, 1780)

= ciliata (Fabricius, 1794) preocc.

= bimaculata (Meigen, 1826)

Hydrotaea floccosa Macquart, 1835

= armipes auct. nec (Fallén, 1825)

Hydrotaea ignava (Harrris, 1780)

= leucostoma (Wiedemann, 1817)

Hydrotaea irritans (Fallén, 1823)

Hydrotaea meridionalis Portschinsky, 1882

Hydrotaea meteorica (Linnaeus, 1758)

Hydrotaea militaris (Meigen, 1826)

Hydrotaea nidicola Malloch, 1925

Hydrotaea palaestrica (Meigen, 1826)

Hydrotaea pandellei Stein, 1899

Hydrotaea parva Meade, 1889

Hydrotaea pellucens Portschinsky, 1879

Hydrotaea pilipes Stein, 1903

Hydrotaea pilitibia Stein, 1916

Hydrotaea ringdabli Stein, 1916

Hydrotaea scambus (Zetterstedt, 1838)

Hydrotaea similis Meade, 1887 
Hydrotaea tuberculata Rondani, 1866

Hydrotaea velutina Robineau-Desvoidy, 1830

POTAMIA Robineau-Desvoidy, 1830

= Dendrophaonia Malloch, 1923

Potamia littoralis Robineau-Desvoidy, 1830

= querceti (Bouché, 1834)

THRICOPS Rondani, 1856

= Alleostylus Schnabl, 1888

Thricops aculeipes (Zetterstedt, 1838)

Thricops albibasalis (Zetterstedt, 1849)

= sudeticus auct. nec (Schnabl, 1888)

Thricops coquilletti (Malloch, 1920)

Thricops cunctans (Meigen, 1826)

= hirsutulus auct. nec (Zetterstedt, 1838)

Thricops diaphanus (Wiedemann, 1817)

Thricops foveolatus (Zetterstedt, 1845)

Thricops genarum (Zetterstedt, 1838)

= sundewalli (Zetterstedt, 1845)

Thricops hirtulus (Zetterstedt, 1838)

= subrostratus (Zetterstedt, 1845)

Thricops innocuus (Zetterstedt, 1838)

Thricops lividiventris (Zetterstedt, 1845)

Thricops longipes (Zetterstedt, 1845)

Thricops nigrifrons (Robineau-Desvoidy, 1830)

Thricops nigritellus (Zetterstedt, 1838)

Thricops rostratus (Meade, 1882)

Thricops rufisquamus (Schnabl, 1915)

= penicillatus (Ringdahl, 1926)

Thricops semicinereus (Wiedemann, 1817)

Thricops separ (Zetterstedt, 1845)

Thricops simplex (Wiedemann, 1817)

tribe Reinwardtiini Brauer \& Bergenstamm, 1889

MUSCINA Robineau-Desvoidy, 1830

Muscina angustifrons (Loew, 1858)

Muscina levida (Harris, 1780)

= assimilis (Fallén, 1823)

Muscina pascuorum (Meigen, 1826)

Muscina prolapsa (Harris, 1780)

= pabulorum (Fallén, 1817)

Muscina stabulans (Fallén, 1817)

MUSCINAE Latreille, 1802

tribe Muscini Latreille, 1802

EUDASYPHORA Townsend, 1911 
Eudasyphora cyanicolor (Zetterstedt, 1845)

Eudasyphora zimini (Hennig, 1963)

MESEMBRINA Meigen, 1826

= Hypodermodes Townsend, 1912

Mesembrina intermedia Zetterstedt, 1848?

Mesembrina meridiana (Linnaeus, 1758)

Mesembrina mystacea (Linnaeus, 1758)

Mesembrina resplendens Wahlberg, 1844

MORELLIA Robineau-Desvoidy, 1830

sg. Morellia Robineau-Desvoidy, 1830

Morellia aenescens Robineau-Desvoidy, 1830

Morellia hortorum (Fallén, 1817)

Morellia podagrica (Loew, 1857)

sg. Ziminiella Nihei \& Carvalho, 2007

Morellia simplex (Loew, 1857)

MUSCA Linnaeus, 1758

Musca autumnalis De Geer, 1776

= corvina Fabricius, 1781

Musca domestica Linnaeus, 1758

Musca tempestiva Fallén, 1817

NEOMYIA Walker, 1859

= Orthellia Robineau-Desvoidy, 1863

Neomyia cornicina (Fabricius, 1781)

= caesarion (Meigen, 1826)

= fennica (Frey, 1909)

Neomyia viridescens (Robineau-Desvoidy, 1830)

= cornicina auct. nec (Fabricius, 1781)

POLIETES Rondani, 1866

Polietes domitor (Harris, 1780)

= albolineatus (Fallén, 1823)

Polietes lardarius (Fabricius, 1781)

Polietes nigrolimbatus (von Bonsdorff, 1866)

Polietes steinii (Ringdahl, 1913)

PYRELLIA Robineau-Desvoidy, 1830

Pyrellia vivida Robineau-Desvoidy, 1830

= cadaverina auct. nec (Linnaeus, 1758)

tribe Stomoxyini Meigen, 1824

HAEMATOBIA Le Peletier \& Serville, 1828

= Lyperosia Rondani, 1856

Haematobia irritans (Linnaeus, 1758)

HAEMATOBOSCA Bezzi, 1907

Haematobosca alcis Snow, 1891

$=$ crassipalpis (Ringdahl, 1920) 
Haematobosca stimulans (Meigen, 1824)

STOMOXYS Geoffroy, 1762

Stomoxys calcitrans (Linnaeus, 1758)

MYDAEINAE Verrall, 1888

GRAPHOMYA Robineau-Desvoidy, 1830

Graphomya maculata (Scopoli, 1763)

Graphomya minor Robineau-Desvoidy, 1830

GYMNODIA Robineau-Desvoidy, 1863

= Brontaea Kowarz, 1873

Gymnodia humilis (Zetterstedt, 1860)

\section{HEBECNEMA Schnabl, 1889}

Hebecnema fumosa (Meigen, 1826)

Hebecnema nigra (Robineau-Desvoidy, 1830)

= vespertina auct. nec (Fallén, 1823)

Hebecnema nigricolor (Fallén, 1825)

Hebecnema umbratica (Meigen, 1826)

Hebecnema vespertina (Fallén, 1823)

= affinis Malloch, 1921

MYDAEA Robineau-Desvoidy, 1830

Mydaea affinis Meade, 1891

= discimana Malloch, 1920

Mydaea ancilla (Meigen, 1826)

Mydaea anicula (Zetterstedt, 1860)

Mydaea corni Scopoli, 1763

= pagana (Fabricius, 1794) preocc.

= scutellaris Robineau-Desvoidy, 1830

Mydaea deserta (Zetterstedt, 1845)

Mydaea detrita (Zetterstedt, 1845)

$=$ electa (Zetterstedt, 1860)

Mydaea humeralis Robineau-Desvoidy, 1830

$=$ tincta (Zetterstedt, 1845)

Mydaea nebulosa (Stein, 1893)

Mydaea obscurella Malloch, 1921

= bengtssoni Ringdahl, 1924

Mydaea orthonevra (Macquart, 1835)

$=$ detrita auct. nec (Zetterstedt, 1845)

Mydaea palpalis Stein, 1916

Mydaea setifemur Ringdahl, 1924

Mydaea sootryeni Ringdahl, 1928

Mydaea urbana (Meigen, 1826)

MYOSPILA Rondani, 1856

Myospila bimaculata (Macquart, 1834)

Myospila meditabunda (Fabricius, 1781) 
OPSOLASIA Coquillett, 1910

Opsolasia orichalcea (Zetterstedt, 1849)

PHAONIINAE Malloch, 1917

tribe Phaoniini Malloch, 1917

HELINA Robineau-Desvoidy, 1830

Helina abdominalis (Zetterstedt, 1846)

Helina allotalla (Meigen, 1830)

Helina annosa (Zetterstedt, 1838)

Helina atricolor (Fallén, 1825)

= denudata (Zetterstedt, 1845)

$=$ denutata emend.

Helina bohemani (Ringdahl, 1916)

Helina celsa (Harris, 1780)

= quadrimaculata (Fallén, 1823) preocc.

= quadrimaculella Hennig, 1957

? Helina ciliata Karl, 1929 (see Notes)

Helina ciliatocosta (Zetterstedt, 1845)

$=$ ciliatocostata emend.

Helina cilipes (Schnabl, 1902)

Helina cinerella (van der Wulp, 1867)

= vanderwulpi (Schnabl, 1888)

= calceata misid.

Helina confinis (Fallén, 1825)

= anceps (Zetterstedt, 1838)

Helina consimilis (Fallén, 1825)

Helina cothurnata (Rondani, 1866)

= obscuripes auct. nec (Zetterstedt, 1845)

Helina daicles (Walker, 1849)

= binotata (Zetterstedt, 1845)

Helina depuncta (Fallén, 1825)

Helina evecta (Harris, 1780)

= lucorum (Fallén, 1823) preocc.

= laetifica (Robineau-Desvoidy, 1830)

Helina flavisquama (Zetterstedt, 1849)

Helina fratercula (Zetterstedt, 1845)

Helina fulvisquama (Zetterstedt, 1845)

Helina impuncta (Fallén, 1825)

Helina latitarsis Ringdahl, 1924

Helina laxifrons (Zetterstedt, 1860)

Helina longicornis (Zetterstedt, 1838)

Helina luteisquama (Zetterstedt, 1845)

Helina maculipennis (Zetterstedt, 1845)

= obscuripes (Zetterstedt, 1845) 
Helina obscurata (Meigen, 1826)

Helina pertusa (Meigen, 1826)

Helina protuberans (Zetterstedt, 1845)

Helina pubiseta (Zetterstedt, 1845)

Helina quadrinotata (Meigen, 1826)

Helina quadrum (Fabricius, 1805)

Helina reversio (Harris, 1780)

= duplicata (Meigen, 1826)

= duplaris auct. nec (Zetterstedt, 1845)

= communis (Robineau-Desvoidy, 1830)

Helina setiventris Ringdahl, 1924

Helina sexmaculata (Preyssler, 1791)

= uliginosa (Fallén, 1825) preocc.

= punctata (Robineau-Desvoidy, 1830)

Helina spinicosta (Zetterstedt, 1845)

Helina squalens (Zetterstedt, 1838)

$=$ borealis (Zetterstedt, 1838)

Helina subvittata (Séguy, 1923)

= roth $i$ Ringdahl, 1939

= marmorata auct. nec (Zetterstedt, 1860)

Helina tetrastigma (Meigen, 1826)

$=$ flagripes $($ Rondani, 1866)

Helina trivittata (Zetterstedt, 1860)

= atripes (Meade, 1889)

Helina veterana (Zetterstedt, 1838)

Helina vicina (Czerny, 1900)

= suecica Ringdahl, 1924

\section{LOPHOSCELES Ringdahl, 1922}

Lophosceles cinereiventris (Zetterstedt, 1845)

= cristata (Zetterstedt, 1845)

Lophosceles frenatus (Holmgren, 1872)

Lophosceles hians (Zetterstedt, 1838)

Lophosceles mutatus (Fallén, 1825)

PHAONIA Robineau-Desvoidy, 1830

= Wablgrenia Ringdahl, 1929

= Dialytina Ringdahl, 1945

Phaonia aeneiventris (Zetterstedt, 1845)

= cinctinervis (Zetterstedt, 1860)

Phaonia alpicola (Zetterstedt, 1845)

Phaonia amabilis (Meigen, 1826)

Phaonia amicula Villeneuve, 1922 (see Notes)

Phaonia angelicae (Scopoli, 1763)

= basalis (Zetterstedt, 1838) 
Phaonia angulicornis (Zetterstedt, 1838)

= erinacea (Fallén, 1824)

Phaonia apicalis Stein, 1914

Phaonia atriceps (Loew, 1858)

Phaonia atrocyanea Ringdahl, 1916

Phaonia canescens Stein, 1916

Phaonia consobrina (Zetterstedt, 1838)

Phaonia czernyi Hennig, 1963

= steinii Czerny, 1900 preocc.

Phaonia errans (Meigen, 1826)

$=$ erratica $($ Fallén, 1825) preocc.

Phaonia erronea (Schnabl, 1887)

Phaonia falleni (Michelsen, 1977)

= vagans (Fallén, 1825) preocc.

Phaonia fugax Tiensuu, 1946

Phaonia fuscata (Fallén, 1825)

Phaonia gobertii (Mik, 1881)

Phaonia gracilis Stein, 1916

Phaonia grandaeva (Zetterstedt, 1845)

Phaonia halterata (Stein, 1893)

Phaonia hybrida (Schnabl, 1888)

Phaonia incana (Wiedemann, 1817)

Phaonia jaroschewskii (Schnabl, 1888)

= crinipes Stein, 1913

Phaonia kowarzii (Schnabl, 1886)

= fulvicornis Tiensuu, 1936

Phaonia laeta (Fallén, 1823)

Phaonia latipalpis Schnabl, 1911

= umbraticola d'Assis-Fonseca, 1957

Phaonia longicornis Stein, 1916

Phaonia lugubris (Meigen, 1826)

$=$ morio $($ Zetterstedt, 1845)

Phaonia magnicornis (Zetterstedt, 1845)

Phaonia meigeni Pont, 1986

= lugubris auct. nec (Meigen, 1826)

Phaonia mystica (Meigen, 1826)

= vittifera (Zetterstedt, 1845)

Phaonia nymphaearum (Robineau-Desvoidy, 1830)

= nitida (Macquart, 1835)

Phaonia pallida (Fabricius, 1787)

Phaonia pallidisquama (Zetterstedt, 1849)

Phaonia palpata (Stein, 1897)

Phaonia perdita (Meigen, 1830)

Phaonia pratensis (Robineau-Desvoidy, 1830) 
= laeta auct. nec (Fallén, 1923)

Phaonia rufipalpis (Macquart, 1835)

Phaonia rufiventris (Scopoli, 1763)

= populi (Meigen, 1826)

Phaonia serva (Meigen, 1826)

Phaonia steinii (Strobl, 1898)

Phaonia subfuscinervis (Zetterstedt, 1838)

Phaonia subventa (Harris, 1780)

= variegata (Meigen, 1826)

Phaonia taigensis Zinovjev, 1987

Phaonia tiefii Schnabl, 1888

Phaonia trimaculata (Bouché)

= servaeformis Ringdahl, 1916

Phaonia tuguriorum (Scopoli, 1763)

= signata (Meigen, 1826)

Phaonia valida (Harris, 1780)

= viarum $($ Robineau-Desvoidy, 1830)

= erratica auct. nec (Fallén, 1825)

Phaonia villana Robineau-Desvoidy, 1830

= mystica auct. nec (Meigen, 1826)

Phaonia vivida (Rondani, 1870)

= austriaca (Czerny, 1900)

Phaonia wablbergi Ringdahl, 1930

Phaonia zugmayeriae (Schnabl, 1888)

?= humeralis (Zetterstedt, 1845)

= humerella (Stein, 1900)

\section{Excluded species}

Coenosia femoralis (Robineau-Desvoidy, 1830) misidentified Fannia barbata (Stein, 1892) not found within present borders Fannia lineata (Stein, 1895) not found within present borders Fannia nigra Malloch, 1910 not found within present borders Hydrotaea glabricula (Fallén, 1825)

Limnophora pollinifrons Stein, 1916 not found within present borders

Phaonia bitincta (Rondani, 1866) misidentified

Scathophaga calida Haliday in Curtis, 1832 not found within present borders

Spilogona acrostichalis (Stein, 1916)

Spilogona norvegica (Ringdahl, 1932)

Spilogona obscuripennis (Stein, 1916) not found within present borders

Spilogona septemnotata (Zetterstedt, 1845) misidentified

Spilogona setigera (Stein, 1907)

Spilogona spininervis (Villeneuve, 1922) 


\section{Notes}

Coenosia comita (Huckett, 1936). Collected only from Salla. The type locality of C. ovulivera Tiensuu, Vuorijärvi, is now Russian territory (Tiensuu 1938). A second specimen, also taken in 1936 by R. Krogerus (in MZH), bears only the label 'Salla' and probably also originates from the vicinity of Vuorijärvi, but it is possible that it was collected from the Finnish part of Salla, a municipality split between Russia and Finland in 1944.

Helina ciliata Karl, 1929. Ringdahl (1952), quoting "Tiensuu's papers", lists this species from Finland. We could not locate the original record in Tiensuu's published works or among the available collection specimens. It seems possible Ringdahl make a mistake in his tables. The species is also listed as Finnish by Hackman (1980), again without any details.

Limnophora pandellei Séguy, 1923. Only females have been found in Finland. The identification remains slightly doubtful (Kahanpää 2013).

Norellia tipularia (Fabricius, 1794). This species was first recorded from Finland by Winqvist (2011). Šifner $(2003,2008)$ considers $N$. tipularia a nomen dubium and probably synonymous with $N$. spinipes Meigen, but Ozerov and Krivosheina (2011) disagree and provide a detailed diagnosis.

Parallelomma paridis Hering, 1923. This name was synonymized with $P$. vittatum (Meigen) by Šifner $(1978)$. Ozerov $(2010,2014)$ examined the types of $P$. paridis and upheld the synonymy. Not everyone agrees with this synonymy (Nelson 1990). Finnish CO1 DNA fingerprints (Kahanpää, unpublished) of larvae on Paris (Liliaceae) and adults caught among Dactylorhiza (Orchidaceae) suggest that two species are involved.

Phaonia amicula Villeneuve, 1922. New to Finland. Recorded as Phaonia sp. nr. halterata by Winqvist (2011): Ab: Turku, Ruissalo (67093:32336), 3.vii.2008, leg K. Winqvist, 1 male.

\section{Acknowledgements}

I would like to express my appreciation to Dr. Verner Michelsen for his constructive feedback and for identifying the first Finnish specimen of Phaonia amicula. Mr. Kaj Winqvist kindly allowed us to include his record of $P$. amicula in this paper.

\section{References}

Hackman W (1956) The Scathophagidae (Dipt.) of Eastern Fennoscandia. Fauna fennica 2: 1-66. Hackman W (1980) A Check List of the Finnish Diptera. Notulae entomologicae 60: 17-48, $117-162$.

Kahanpää J (2013) Viisikymmentä Suomelle uutta kärpäslajia (Diptera: Brachycera). Sahlbergia 19(1-2): 63-71. 
Kahanpää J, Haarto A (2013) Notes on Fanniidae (Diptera) of Finland, with a description of the female of Fannia stigi Rognes, 1982. Entomologica fennica 24: 179-185.

Kutty SN, Pape T, Wiegmann BM, Meier R (2010) Molecular phylogeny of the Calyptratae (Diptera: Cyclorrhapha) with an emphasis on the superfamily Oestroidea and the position of Mystacinobiidae and McAlpine's fly. Systematic Entomology 35(4): 614-635. doi: 10.1111/j.1365-3113.2010.00536.x

Nelson JM (1990) Observations on the biology and status of British dung-flies of the genus Parallelomma Becker (Dipt., Scathophagidae). Entomologist's Monthly Magazine 126: 187-189.

Nirmala X, Hypša V, Žurovec M (2001) Molecular phylogeny of Calyptratae (Diptera: Brachycera): the evolution of $18 \mathrm{~S}$ and $16 \mathrm{~S}$ ribosomal rDNAs in higher dipterans and their use in phylogenetic inference. Insect Molecular Biology 10(5): 475-485.

Ozerov AL (2010) Flies of the genus Parallelomma Becker in Strobl, 1894 (Diptera, Scathophagidae) of Russia. Russian Entomological Journal 18[2009](4): 309-317.

Ozerov A (2014) The Primary Types of Scathophagidae (Diptera) in the Museum für Naturkunde Berlin, and Senckenberg Deutsches Entomologisches Institut, Germany. Zoosystematics and Evolution 90(1): 33-43. doi: 10.3897/zse.90.7121

Ozerov AL, Krivosheina MG (2011) The first record of Norellia tipularia (Fabricius, 1794) (Diptera: Scathophagidae) for the fauna of Russia. Russian Entomological Journal 20(1): 101-103.

Pape T, Blagoderov V, Mostovski MB (2011) Order Diptera Linnaeus, 1758. In: Zhang Z-Q (Ed) Animal biodiversity: An outline of higher-level classification and survey of taxonomic richness. Zootaxa 3148: 222-229. http://www.mapress.com/zootaxa/2011/f/zt03148p229.pdf

Ringdahl O (1952) Catalogus Insectorum Sueciae. XI. Diptera Cyclorrhapha: Muscaria Schizometopa. Opuscula entomologica 17: 129-186.

Šifner F (1978) La révision synonymique des espèces du genre Americina Malloch, 1923 (Diptera, Scatophagidae). In: Országh I (Ed) Proccedings of the IVth Meeting of Czechoslovak Dipterists. Dipterologica bohemoslovaca 1. Veda, Bratislava, 283-302.

Šifner F (2003) The family Scathophagidae (Diptera) of the Czech and Slovak republics (with notes on selected Palaearctic taxa). Acta Musei Nationalis Pragae, Series B, Historia Naturalis 59(1-2): 1-90.

Šifner F (2008) A catalogue of the Scathophagidae (Diptera) of the Palaearctic region, with notes on their taxonomy and faunistics. Acta entomologica Musei Nationalis Pragae 48(1): 111-196. http://www.aemnp.eu/pdf/48_1/48_1_111.pdf

Tiensuu L (1935) Die bisher aus Finnland bekannten Musciden. Acta Societatis pro Fauna et Flora Fennica 58(4): 1-56.

Tiensuu L (1938) Beiträge zur Kenntnis der Musciden (Dipt.) Finnlands. Annales entomologici fennici 4(1): 21-33.

Tiensuu L (1939) Beiträge zur Kenntnis der Musciden (Dipt.) Finnlands. 2. Annales entomologici fennici 5(4): 241-255.

Winqvist K (2011) Ruissalon kärpäsistä (Diptera, Brachycera). w-album 11: 3-23. http://org. utu.fi/harrastus/TEKS/w-album/w_album_11.pdf 